\title{
Costos de producción en la cría de pollos de engorde*-
}

\author{
Orozco Campo, Rafael $* *$ \\ Meleán Romero, Rosana*** \\ Rodríguez Medina, Guillermo**** \\ ** Econ. Mg. en Gerencia de Empresas. Prof. Titular. FCES, Investigador del CEE. E- \\ mail: Rafaoro50@hotmail.com \\ *** Lic. en Administración. Becaria Académica CEE. rosanamelean@cantv.net \\ **** Dr. En Ciencias, Mención Gerencia. Prof. Titular. FCES, Investigador del CEE. E- \\ mail: grodriguezm@cantv.net
}

\section{Resumen}

La presente investigación se centra en el análisis de los costos de producción en la cría de Pollos de Engorde en las siguientes empresas ubicadas en los Municipios Mara, La Cañada de Urdaneta y Jesús Enrique Lossada del Estado Zulia en Venezuela: Pinpollo, Vilva, Avícola de Occidente, Protinal, Proave, Souto, y Criazuca. Se realiza un esbozo de la política agrícola gubernamental venezolana; se caracteriza el proceso productivo, específicamente el de engorde de pollo de las empresas mencionadas, se analizan y discuten las estructuras de costos de dichas empresas. La investigación es de tipo bibliográfica-empírica, de carácter descriptivo, bajo un enfoque no experimental. Se concluye que: los altos costos de los rubros de alimentación, que representa el $71 \%$ del total de los costos de producción y un 13\% correspondientes a la adquisición de los pollitos bebés como insumos para iniciar el proceso de engorde de las aves, constituyen las bases de la integración vertical de la industria avícola; en esta integración, las empresas grandes ejercen control sobre empresas de menor tamaño, en este caso las granjas criadoras de los pollos, quienes aceptan las condiciones de crianza, higiene y alimentación entre otras impuestas por la empresa que controla la red.

Palabras clave: Pollos de engorde, Costos de producción, Integración Vertical, Producción Avícola.

\section{Production Costs in Rising of Fattening Chickens}

\section{Abstract}

This research centered its analysis on the production costs of broiler chicken in the 
following companies located in Municipios Mara, La Cañada de Urdaneta and Jesus Enrique Lossada, Zulia State, Venezuela: Pinpollo, Vilva, Avicola de Occidente, Protinal, Proave, Souto and Criazuca. A study was made of the Venezuelan governmental political agricultural policy, and the productive policy and cost structures of each company were analyzed. The research was biographical-empirical, descriptive and non-experimental. The conclusions were that production costs represented $71 \%$ of the total cost, the purchase of the baby chicks at the beginning of the productive cycle, was $13 \%$ of the cost and constituted the basis of the vertical integration in the poultry industry. In the industry, the larger companies control the smaller ones, in this case the chicken farms must accept the conditions imposed as to hygiene, feeding, sanitation etc imposed by the companies that control the system.

Key words: Broiler chicken, production costs, vertical integration, poultry production.

Recibido: 04-04-13. Aceptado: 04-10-13

\section{Introducción}

La difícil situación que atraviesa Venezuela al igual que muchos países de América Latina, la hace enfrentar grandes desafíos en materia micro y macroeconómica. La apertura de la economía al capital internacional, la devaluación de nuestro signo monetario, la inflación, la pesada deuda externa y la diatriba política, han repercutido en el crecimiento de nuestro producto interno y generado una grave crisis que debe superar el país.

El aporte anual de la avicultura venezolana a los 24 millones de habitantes del país es elevado, en lo que respecta a pollos, gallinas ponedoras y huevos, renglones éstos que proveen toda la proteína avícola que consume el venezolano.

La producción avícola nacional está dedicada al mercado interno llegando a tener según información suministrada en la primera fase del censo agrícola (1997) el 70\% del mercado de las carnes, desplazando a las carnes bovinas que habían sido el alimento cárnico por excelencia en el país. Los avicultores venezolanos saben que su quehacer más importante es convertirse en productores cada vez más eficientes, capaces de abastecer de una proteína barata y de alta calidad como los huevos y la carne de pollo a toda la población y en especial a la población de extrema pobreza.

El estado Zulia, en particular los municipios Mara, la Cañada de Urdaneta y Jesús 
Enrique Losada son considerados grandes productores avícolas, según estimaciones del último censo agrícola (1997), esta entidad contaba con aproximadamente 5.676 explotaciones avícolas con su producción anual estimada de 70 millones de pollos de engorde anuales, lo que representa más del $20 \%$ de la producción nacional, razón por la cual constituyen los municipios en los cuales se encuentran ubicadas las granjas a estudiar en esta investigación, cuyos propietarios han asumido el riesgo de invertir y gerenciar recursos en un sector de la economía que está sometido a exigencias constantes del distribuidor, consumidor y del mismo Estado.

El conocimiento de los elementos de los costos de producción y su respectiva evolución, constituyen elementos importantes tanto para los productores como para el Estado; para los primeros por la relevancia de esta variable (los costos) en la toma de decisiones gerenciales y para el segundo por ser una herramienta fundamental en la adopción de políticas gubernamentales.

La investigación que aquí se realiza no pretende determinar si han existido o no incrementos en los costos de producción del pollo, sino analizar y discutir el comportamiento que han tenido los elementos que conforman la estructura de costos de siete granjas pertenecientes a empresas diferentes; asimismo, se pretende caracterizar el proceso productivo del pollo de engorde, es decir, identificarlo y contrastarlo con nuevos conceptos o enfoques contemporáneos.

\section{Política agrícola venezolana}

El contexto venezolano, sin duda ha sido muy difícil en los últimos años. El paro empresarial iniciado a finales del 2001 y que prosiguió en el 2002, llegando a producir un Golpe de Estado el 11 de abril del mismo año, al que se suma un paro petrolero y consecutivas protestas, dejaron consecuencias devastadoras en la economía venezolana. El control de cambio decretado por el gobierno nacional, en febrero de 2003 para regular y controlar la compra y venta de divisas en el país; el control de precios, decretado en febrero para regular los precios de los productos de primera necesidad, entre los cuales se encuentran las carnes de: res, pollo, pavo, gallina, ovinos, caprinos y porcinos, así como los dos meses de inactividad (diciembre 2002 y enero 2003); fueron sin duda acontecimientos que han repercutido en las actividades habituales de las empresas venezolanas y con ello en la rentabilidad de las mismas, sin obviar las cuantiosas pérdidas ocasionadas al país.

Otros argumentos empresariales son: la pérdida del poder adquisitivo de los venezolanos 
y las restricciones existentes para las importaciones, por los rigurosos trámites para la obtenci ón de divisas y por la cantidad de obligaciones fiscales y parafiscales exigidas por la Comisión de Administración de Divisas (Cadivi, 2003), a causa de las poca cultura de pago de los impuestos; ante esta situación, en junio de 2002 el gobierno otorgó licencias para importar 150 mil toneladas de maíz, sólo después de asegurarse de que la producción criolla de este rubro se había consumido casi por completo; al respecto, Efrén Andrades titular del Ministerio de Agricultura y Tierras (MAT) citado por Barreiro (2003) solicitó a Cadivi que entre los meses de septiembre y octubre de 2003 se entregaran 180 millones de dólares para la importación de alimentos relacionados con las fiestas navideñas, asimismo, el MAT puso en marcha un plan especial para aumentar la producción de proteína animal. La idea es congelar el producto para que el mercado esté abastecido, llegando a congelar 15 mil toneladas de pollo, 5 mil de cerdo, 2.500 de pavo y 1.500 de gallina; tal situación se tornó en acaparamiento, lo que llevó al gobierno a importar grandes cantidades de pollo entre otros alimentos para abastecer de éstos productos a la población.

El estudio de la avicultura y en particular la producción de pollos de engorde reviste gran importancia debido a que es una actividad económica generadora de empleo, produce alimentos e insumos para el Sector Agro- Industrial. Dentro de este contexto adquieren notable importancia los renglones pollos frescos, huevos y otros rubros con valor agregado que son de carácter vital para la población, ya que forman parte de la canasta de alimentos básicos del venezolano.

Los esfuerzos del gobierno, se deben orientar más hacia la producción nacional, generando mayores fuentes de empleo para la población, puesto que si continúan las importaciones de alimentos se incrementarán los problemas de desempleo en el país, y como establece Rafael Alfonso, presidente de la Cámara Venezolana de la Industria y Alimentos (Cavidea), citado por Hernández (2003), la solución para garantizar la alimentación no es importar productos terminados; sin embargo, el gobierno adoptó esta medida dado el acaparamiento de productos por parte de las empresas del sector alimenticio nacional, durante el paro empresarial y petrolero.

Los grandes problemas que existen hoy en el sector alimenticio, específicamente en el sector avícola nacional, no son cosa fácil de solventar; el Ejecutivo Nacional, regula el precio del pollo el cual no es acatado por los productores, quienes argumentando costos más elevados, aprovechan la situación para especular, siendo los más afectados los consumidores finales. De acuerdo a Perozo (2003:2-4), el gobierno importó 600 toneladas de pollo de Brasil para hacer frente a la escasez coyuntural (producto del acaparamiento 
realizado por las grandes empresas opuestas al gobierno constitucional) y garantizar la regulación del precio. Así pues, se evidencia la existencia de esta controversia; para el gobierno, la prioridad suprema es garantizar la seguridad alimentaria de la población, ofreciendo precios accesible; sin embargo, para los productores la prioridad es cubrir sus costos de producción y obtener un margen de ganancia por la actividad que realizan, alegando que los precios regulados por el gobierno no cubren sus costos de producción. A este respecto, la Constitución de la República Bolivariana de Venezuela, establece en su artículo 305 que el Estado debe garantizar la seguridad alimentaria de la población que consiste en la disponibilidad suficiente y estable de alimentos en el ámbito nacional y el acceso oportuno y permanente a ellos por parte del público consumidor.

La definici ón aprobada por la Organización de las Naciones Unidas para la Agricultura y la Alimentación, FAO1 (FAO, 2003a), plantea que existe seguridad alimentaria cuando todas las personas tienen en todo momento acceso físico y económico a comida suficiente. Venezuela depende en un alto porcentaje de la importación, circunstancia que se vió agravada en los meses del paro empresarial por problemas de transporte $y$ acaparamiento, entre otros, donde el gobierno debió asumir y ejecutar políticas para contrarrestar el desabastecimiento de alimentos en la población. Para ello, Venezuela se insertó en las políticas de la Organización de las Naciones Unidas para la Agricultura y la Alimentación (FAO, 2003b), y establece el Programa Especial para la Seguridad Alimentaria (PESA), el cual tiene como objetivo ayudar a los países en vías del desarrollo, especialmente los países de bajos ingresos con déficit de alimentos (PBIDA) para mejorar su seguridad alimentaria.

El plan PESA, apoyado por los Ministerios de Producción y Comercio, y de Agricultura y Tierras tiene como finalidad transformar y diversificar la producción agropecuaria de los pequeños productores y mejorar la seguridad alimentaria, tanto rural como urbana de la población (FAO, 2003b). Tal es el caso de los Mercados de Alimentos (Mercal) construidos por el gobierno nacional e insertados en el Plan PESA para proveer alimentos de primera necesidad, a un costo más bajo para la población de escasos recursos.

Según informaciones de Venpres, el presidente de la Corporación Venezolana Agrícola (CVA) Freddy Gil, señaló que para finales del 2003, se invertirían 170 millardos de bolívares para programas específicos, así como, un plan avícola y comercializadoras de insumos agropecuarios; de igual manera, sostuvo que con el Fondo Intergubernamental para la Descentralización (Fides), se encuentran próximos a efectuar un convenio para trabajar en la formación y constitución de cooperativas, las cuales serían de producción de alimentos y comercializadoras de productos (González, 2003). 
En lo que respecta al consumo de productos extranjeros, los cálculos de la Comisión Presidencial Agrícola indican que Venezuela durante el 2001 importó un millón cuatrocientos setenta y ocho (1.478.000) toneladas en productos agrícolas, algunos de ellos considerados tan criollos como las caraotas, tomates, azúcar, harina de yuca, aceites, entre otros.

Una de las razones de la importación de más del $60 \%$ de los alimentos que consumen los venezolanos, es que la producción nacional no satisface la demanda, pero no sólo el déficit la impulsa, sino que los países líderes en la producción de bienes agrícolas compiten por colocar sus productos subsidiados en nuestros mercados y los importadores nacionales luchan por mantener un negocio de importación de más de un mil millones de dólares mensuales, de tal manera que es obvio que un aumento en la producción agrícola nacional afectará tan suculento negocio.

Por otra parte, el gobierno a través de la Ley de Mercadeo Agrícola, aprobada en la Habilitante, trata de restringir la anterior situación, y basado en dicha Ley, el Ministerio de Agricultura y Tierras estima la demanda nacional de un rubro junto con los productores agrícolas pertenecientes a la Federación de Productores del Agro (FedeAgro) y los Agroindustriales. En función de ese cálculo se determina la cantidad que se traerá de afuera, luego que la producción venezolana tenga su colocación garantizada (Carvajal, 2002: 5).

En cuanto a las políticas gubernamentales, si el gobierno en lugar de aplicar una política de subsidio a la producción nacional, decide por el contrario, aplicar la máxima neoliberal de una economía de libre mercado ¿Qué posibilidad real tendría el sector agropecuario criollo de competir ventajosamente en el mercado mundial?. La repuesta es ninguna, porque nuestros productos no son competitivos; aplicar el libre mercado a los productos agrícolas implicaría subsidiar a agricultores estadounidenses y europeos. Germán Briceño, Ingeniero Agrónomo asesor de FedeAgro, opinó sobre la factibilidad de que Venezuela se abra totalmente al mercado mundial: "Reto a cualquiera a que trate de colocar sus productos en USA, que es el campeón del Neo-Liberalismo", y señaló que el gobierno norteño no sólo dispone de sofisticados filtros arancelarios, sanitarios, ambientales, entre otros, sino que subsidia su agricultura con millardos de dólares (Carvajal, 2002).

La diferencia fundamental la pone la tecnología; Venezuela carece de máquinas y sistemas operativos eficientes que permitan elevar la productividad por hectárea o litros, condición básica para ser competitivo. Mientras se tenga como promedio una producción 
de 1800 kilogramos de maíz por hectárea, vacas con 5 litros por ordeño, difícilmente se puede competir; la diferencia no sólo está en tener hectáreas o silos, hoy en día existe la Ingeniería Genética, que ofrece mejoras en los cultivos y la cría de animales, haciéndolos más productivos y resistentes a plagas y enfermedades. Ahora bien, por lo antes expuesto, cabe preguntarse ¿No sería mejor entonces, traer del exterior los alimentos si son más económicos en el mercado internacional?, la respuesta sería que ningún país pone en manos del resto del mundo la totalidad de su seguridad alimentaria; por muy estériles que sean sus tierras, siempre hará un esfuerzo supremo para suplir en algún porcentaje el consumo nacional.

\section{Proceso productivo en empresas del sector avícola: Pollos de engorde}

Para definir el proceso productivo es importante conceptualizar el término producir, que según el diccionario pequeño Larousse (1997: 825) significa fabricar, transformar materias primas en manufacturadas. Por su parte Davis, et al (2001: 7), definen el proceso de fabricación o como lo denominan Fernández, et al (2003:9) proceso productivo; como el conjunto de actividades mediante las cuales uno o varios factores productivos (tierra, capital, tecnología y fuerza de trabajo) se transforman en productos; dicha transformación crea riqueza y añade valor a los insumos o componentes de la empresa; a medida que avance el producto a lo largo del sistema de producción mayor será el valor del adquirido.

Para Chase, et al (2000:6) un sistema de producción es simplemente aquel que utiliza recursos operacionales (máquinas, personas, herramientas o un complejo sistema de producción) para transformar las entradas o insumos en algún tipo de resultado deseado: bienes y servicios, mientras que Sipper y Bulfin (1998:7), agregan que dichas salidas deben tener un valor inherente, definiéndolo en su acepción más simple como cualquier actividad que produzca algo. Al respecto, Rodríguez, et al (2002: 137), establecen que el proceso productivo está referido a la utilización de recursos operacionales que permiten transformar la materia prima en un resultado deseado, que bien pudiera ser un producto terminado.

Un Sistema de Producción atraviesa por varias etapas que van desde su nacimiento hasta su madurez. Chase, et al (2000:11) dividen estas etapas de la manera siguiente: Nacimiento del sistema, diseño del producto y selección del proceso, diseño del sistema, administración de la cadena de suministros y revisión del sistema.

En el proceso productivo, también es importante el término Productividad, como un 
indicador de eficiencia de las operaciones de la planta, que implica una relación Producto Obtenido - Insumo Utilizado. Para Horngren, et al (1996:773) la productividad mide la relación entre insumos reales (en cantidades y en costos) y la producción real alcanzada; mientras menor sea la cantidad de los insumos para una serie determinada de producción o mientras mayor sea la producción para una serie determinada de insumos, mayor es el nivel de Productividad.

Para Gamboa y Naveda (1999) la productividad; establece la proporción (cociente) entre el volumen de la producción y la cantidad o valor de los insumos utilizados. Desde el punto de vista físico, sólo puede calcularse en forma parcial, mientras que en unidades monetarias puede medirse tanto parcial como globalmente. En su sentido más amplio la Productividad se define como Outputs/ Inputs, es decir, es el resultado de dividir el total de factores de salida, como bienes, entre los factores de entrada, como recursos (Oficina Internacional del Trabajo, citada por García, 1995: 16), o como es definida simplemente por Davis, et al (2001: 106) es la eficiencia de un proceso.

El Proceso Productivo de la Industria Avícola, involucra también dos elementos importantes referidos a la Producción Agrícola Vegetal y Animal. La primera está compuesta en su mayor parte por cereales, básicamente maíz, sorgo y soya importada, ya que la producción nacional de esta última es muy limitada; luego, la segunda, una vez que la Producción Agrícola esté preparada, se inicia el proceso de transformación industrial, que es ejecutada por las plantas de alimentos balanceados para animales, que se considera el eslabón más importante dentro de esta cadena, puesto que el producto final que allí se origina resulta ser un elemento insustituible en la Producción Avícola en general. Los alimentos balanceados participan en otros circuitos de Producción Animal (cerdos, bovinos y otros), pero la mayor cantidad del producto se destina a aves y cerdos. Según Velasco (1997: 48), las industrias de alimentos balanceados han jugado un papel preponderante en el desarrollo de la Industria Avícola, especialmente en la forma de integración que se da entre un gran número de granjeros y los complejos agroindustriales, ya que los mismos han sido los responsables de la asistencia técnica de la investigación en el área Avícola.

Es pertinente destacar que los alimentos y medicinas necesarios para el engorde de las aves provienen en buena medida de grandes empresas como Protinal del Zulia, Gramoven, Purina de Occidente, Proave, Vilva y Alinteca, así como también Souto y Pimpollo entre otras. Estas compañías son las que cubren en toda su magnitud las necesidades de alimentación de la industria avícola; sin embargo, existen además pequeñas y medianas empresas de alimentos concentrados que han surgido como 
productoras independientes de éstos insumos básicos para la producción avícola, pero que también suministran dichos rubros a granjeros independientes 0 satisfacen deficiencias momentáneas de los grandes complejos.

La Industria de Alimentos Balanceados, ha brindado a los granjeros un sistema de financiamiento y abastecimiento de insumos básicos y equipos necesarios para la producción y en buena medida se ha encargado de la comercialización del producto. Además, gran parte de los centros de beneficios (mataderos) están integrados a las fábricas de alimentos balanceados, que cierran el circuito de financiamiento que se inicia con el suministro de alimento concentrado y los pollitos bebés.

En los párrafos anteriores, se evidencia la integración vertical de la industria avícola; entendida según la Cooperación Económica Asiática (1999:8) como la organización de la industria a lo largo de un número creciente de componentes de la cadena de valor y a la mayor estandarización de la producción a cada etapa del proceso; la integración no necesariamente implica una consolidación financiera de los diferentes componentes de la cadena de valor, ni un incremento en la escala de producción de cada unidad de negocio, en este proceso el objetivo consiste en la constante búsqueda por ejercer control sobre los componentes individuales del proceso de producción avícola.

Según Gamboa, et al (2003:31) la integración vertical de la red tiene como objetivo aumentar el control sobre los proveedores de una empresa o adquirir el dominio sobre ellos. Según las autoras, la integración vertical en redes de proveedores o distribuidores busca asegurar la colaboración de empresas de menor tamaño con la empresa líder en el suministro o en la distribución. En tal sentido, la integración del sector avícola, se presenta de la siguiente manera: las empresas de menor tamaño; representadas por las granjas y las grandes empresas que controlan la red, las cuales serían Protinal del Zulia, Gramoven, Purina de Occidente, Proave, Vilva, Alinteca, Souto y Pimpollo; ellas suministran los pollitos bebés y los alimentos para el proceso de engorde de los pollos; el objetivo es ejercer un control pleno de las granjas que se dedican a la cría de pollos y ponedoras.

Al hablar de integración vertical, Coriat (1995:100) se refiere a las relaciones entre otorgantes (empresa matriz) y subcontratistas. Este autor, plantea que en Japón como en otras partes del mundo, es frecuente que las grandes empresas se esfuercen por reducir sus inversiones en capital fijo, subcontratando algunas actividades con pequeñas $y$ medianas empresas, que en éste caso estaría constituido por las granjas donde se lleva a cabo el proceso cría y engorde de las aves. 
El proceso de engorde de pollos, recibe de las empresas integradas los pollitos bebés y los alimentos principalmente, mientras que las granjas o criadores colocan la mano de obra y adecuan las condiciones higiénicas de las instalaciones para evitar enfermedades en las aves. Es importante desatacar que los honorarios de los criadores se negocian y se basan en la estructura de la industria y las condiciones locales.

Al respecto Asanuma, citado por Coriat (1995:101-102), plantea que existen cuatro proposiciones que permiten caracterizar la empresas matriz y subcontratista: 1) la relación de subcontratismo es de largo plazo, cuya duración está determinada por el ciclo de vida de los productos; 2) es una relación institucionalizada y jerarquizada; 3) es una relación contractualizada, objeto de procedimientos particulares; y 4) la relación favorece e “internaliza” la innovación.

Desde otra perspectiva, en lo que respecta a la selección del sistema productivo, Rodríguez et al. (2002, 139), sostienen que está íntimamente ligado a la estrategia del producto, es decir, según esté enfocada al proceso o al producto, dando origen a los sistemas productivos básicos de la demanda intermitente o de demanda continua; al respecto Buffa y Sarin (1992), citado por Rodríguez et al (2002), argumentan que un sistema productivo para demanda intermitente está confeccionado para fabricar productos a la medida, donde el énfasis de la estrategia del producto es su especificidad, confiabilidad de entrega a tiempo, calidad y flexibilidad; mientras que el sistema de producción continua se caracteriza por elaborar productos estandarizados con altos niveles de producción. En tal sentido, Horngren et al. (1996:99) manifiestan que en el primero se obtienen costos unitarios asignándole éstos a un producto identificable, distinto y para un cliente en específico; mientras que en un sistema por proceso continuo, el costos se obtiene al asignarlo a masas de unidades similares, y luego se calculan los unitarios sobre una base promedio; a menudo, se producen artículos idénticos para su venta en general y no para un cliente en específico, éste es el sistema de costeo utilizado en el proceso de engorde de pollos.

En el Diagrama 1, se observan los factores de producción o factores de costos; necesarios para el proceso de engorde de las aves; en él intervienen la fuerza de trabajo y los medios de producción que transforman un objeto en un producto terminado. El objeto de trasformación está constituido por los pollitos bebés que se someten a un proceso de engorde, dónde se realiza una conversión de alimentos para lograr en las aves un peso de aproximado de 1.8 y $2.1 \mathrm{Kg}$. para posteriormente pasar al proceso de beneficio, en el proceso de engorde de pollos por lo general se producen algunos desechos como pollos 
descartados o muertos.

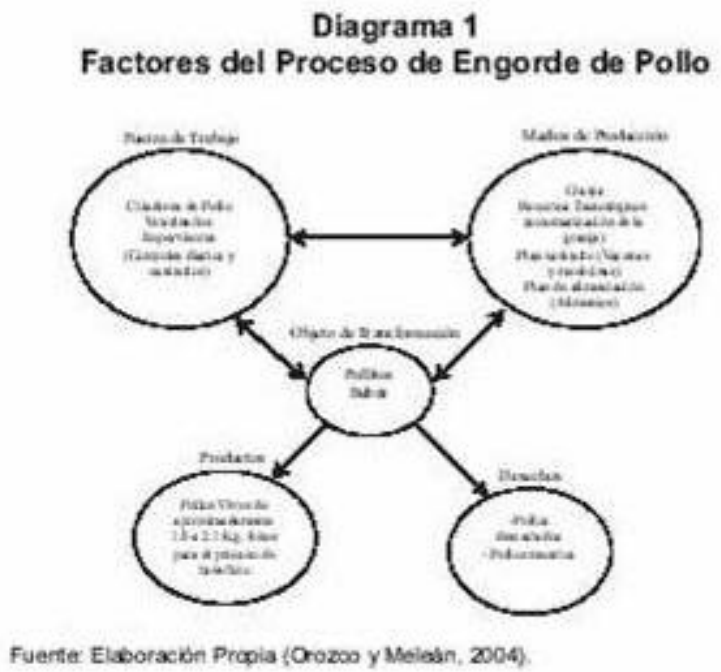

En esta investigación, el análisis estará centrado en el proceso de engorde de pollos (crecimiento y crianza), como una etapa del proceso productivo de pollos en empresas del sector, entendiéndose por ellos, el ave comercializable con edad aproximada de seis semanas y que en la mayoría de los casos su peso vivo es de 1.8 kilogramos; sin embargo, existen variaciones en cuanto al peso final de mercado y la manera cómo se comercializa. En tal sentido, representado gráficamente el proceso de engorde de pollos sería: (Ver Diagrama 2).

El proceso productivo en las industrias avícolas, consta de varias etapas:

La primera etapa comprende el establecimiento de las granjas de progenitores, constituidas por aves nacionales o provenientes del exterior; éstas darán origen a padres que conformarán las granjas de reproductoras.

La segunda etapa, corresponde a la obtención, en las granjas reproductoras de los huevos fértiles que darán origen a los pollitos, los cuales constituyen el insumo kásico para iniciar el proceso de engorde.

La tercera etapa, se refiere al proceso de incubación, que es la última etapa de la cadena para producir el insumo básico (pollo bebé), requerido en las granjas de engorde.

El proceso comienza con la entrada de los insumos necesarios, constituidos en este caso por el lote de pollitos bebés con sus respectivos alimentos, vacunas y medicamentos; posteriormente se ubican en los espacios del galpón destinados para la cría, los cuales deben estar preparados, bajo estrictas condiciones sanitarias y climáticas. El proceso de engorde tiene una duración que promedia los 42 días, tiempo en el cual las aves deben 
alcanzar entre 1.8 a $2.1 \mathrm{Kg}$. de peso vivo; una vez alcanzado este peso, se hace entrega del lote al matadero para su beneficio. Posteriormente, cada granja es sometida a un período de descanso y saneamiento que dura unos 15 días, para prevenir cualquier brote infeccioso, culminado este proceso se inicia uno nuevo.

Diagrama 2

Proceso productivo de pollos de engorde

\begin{tabular}{|c|c|c|}
\hline trstatos & 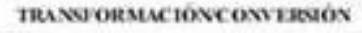 & paovectonkis. \\
\hline $\begin{array}{l}\text { - Pollitos Belds } \\
\text { - Alimerison } \\
\text { - Vacanas y } \\
\text { Medicias }\end{array}$ & 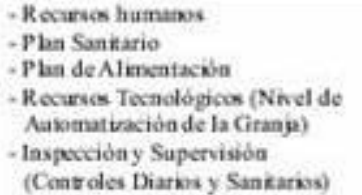 & 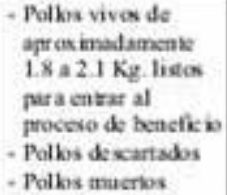 \\
\hline
\end{tabular}

Funte: Elaboradion Propia (Orozeo y Melest, 2004).

Cualquiera que críe pollos debe efectuar una observación detallada de las variaciones en el crecimiento y el consumo de alimentos, tanto en los pollos machos como en las hembras. Estas variaciones incluyen según North y Bell (1993:400): 1) el peso corporal; 2) incrementos semanales del peso corporal; 3) consumo semanal de alimentos; 4) consumo acumulativo de alimentos; 5) conversión semanal de alimentos; y 6) conversión acumulativa de alimentos.

La información contenida en la Tabla 1 representa cifras promedio para buenos lotes (parvadas) de pollos de engorde. Existen variaciones estacionales en estas cifras que pueden reflejar, según los autores mencionados los siguientes hechos:

1. Los pollos no crecen a una tasa uniforme, el crecimiento se inicia lentamente en un período de aceleración, luego aminora antes de la madurez sexual.

2. Los machos crecen más rápido que las hembras; y tendrán un peso predeterminado cuatro días antes de que las hembras lo logren.

3. Los incrementos semanales de peso en lotes uniformes: El peso del lote se incrementa hasta alcanzar un máximo alrededor de la séptima semana, luego decrecen.

4. El consumo de alimentos semanal se incrementa al subir el peso; las aves acrecientan su consumo de alimentos semana tras semana.

5. Los primeros aumentos de peso requieren menos alimentos: La conversión o 
las unidades de alimentos necesarias $(\mathrm{Kg})$ para producir un Kilo de peso, son más bajas en las primeras semanas, después se incrementan por semana. Por ejemplo, un lote uniforme produce un $\mathrm{Kg}$ de aumento de peso en la segunda semana por 1,21 Kg de alimento, pero requiere $2,32 \mathrm{Kg}$ en la séptima semana. para aumentar el mismo peso.

6. Los machos convierten más eficientemente el alimento a carne que las hembras: Un macho que pese $2.01 \mathrm{Kg}$ requiere casi $3,48 \mathrm{Kg}$ de alimento, mientras que una hembra del mismo peso necesita 3,94 Kg. La conversión de alimento es de 1,73 para machos y de 1,89 para hembras.

\section{Mientas más pesado sea el lote completo, mayor es la diferencia en peso de}

Ios sexos: A las dos semanas de edad el macho pesa $107 \%$ del peso de la hembra, a las seis semanas de edad es de $115 \%$.

Debido a que el alimento representa el insumo más caro en la producción del Pollo de Engorde y por la variación en la conversión de alimentos de acuerdo a la edad, sexo y peso del ave, lo mejor es que se comprendan dichas variaciones y sus implicaciones en las ganancias de peso. En la medida que el Pollo de Engorde envejece y se vuelve más grande, el consumo de alimento aumenta y la conversión de alimento disminuye.

El tiempo implica costos en la producción de pollos de engorde. Mientras que el pollo dure más tiempo que el necesario para alcanzar el peso corporal deseado, los costos se elevarán. Como los machos alcanzan un peso deseado varios días antes que las hembras, es preciso registrar el número de días requeridos para alcanzar el peso corporal deseado por sexo. 
Tabla 1

Consumo y conversion alimenticia de alimentios en pollos de engorde (enkg)

\begin{tabular}{|c|c|c|c|c|c|c|c|c|c|c|c|c|c|c|c|c|c|c|}
\hline \multicolumn{7}{|c|}{ Moshos } & \multicolumn{6}{|c|}{ Henters } & \multicolumn{6}{|c|}{ Mntos } \\
\hline & Pros & Wo & \multicolumn{2}{|c|}{$\begin{array}{l}\text { Consuno de } \\
\text { yruntes }\end{array}$} & \multicolumn{2}{|c|}{$\begin{array}{l}\text { Conerida } \\
\text { Aineriteis }\end{array}$} & \multirow{2}{*}{\multicolumn{2}{|c|}{$\begin{array}{l}\text { PVo Wo } \\
\text { find Cresia }\end{array}$}} & \multicolumn{2}{|c|}{$\begin{array}{l}\text { Cossum d } \\
\text { llarios }\end{array}$} & \multicolumn{2}{|c|}{$\begin{array}{l}\text { Cowernibs } \\
\text { Almeticis }\end{array}$} & \multirow{2}{*}{\multicolumn{2}{|c|}{$\begin{array}{l}\text { Pow Who } \\
\text { Finl Gencis }\end{array}$}} & \multicolumn{2}{|c|}{$\begin{array}{l}\text { Conumo ot } \\
\text { sinestes }\end{array}$} & \multicolumn{2}{|c|}{$\begin{array}{l}\text { Convered } \\
\text { Ninertices }\end{array}$} \\
\hline Bal & $\begin{array}{l}\text { Fele } \\
\text { isenes }\end{array}$ & $\begin{array}{l}\text { Cend } \\
\text { Snend }\end{array}$ & tant & enit & Send & turnie: & & & tent & Lent & inom & & & & Sered & insil & Swx & nit \\
\hline 1 & to & Q18 & 118 & DQ & in & th & ast & As & 16 & 10 & all & $1 N$ & (1) & ts: & 012 & 10 & $\Delta$ & 10 \\
\hline 2 & A4 & ts & 18) & 16 & 12 & in & all & 18 & DY & 16 & 12 & 58 & a) & as & AX & in & 18 & 18 \\
\hline 1 & in & 128 & 16 & 18 & 11 & 13 & if & 18 & 14 & in & 111 & 18 & 60 & IX & 16 & 18 & 16 & ix \\
\hline 1 & in & OD & $w$ & 18 & 18 & 18 & 10 & en & 15 & 10 & 13 & 18 & 18 & ix & 40 & 181 & 17 & $|t|$ \\
\hline 1 & 14 & 10 & 14 & 26 & $|1|$ & 19 & 187 & 017 & in & 22 & 24 & 10 & 16 & is & IX & 2N) & W & 19 \\
\hline 1 & 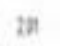 & 18 & 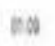 & 24 & 23 & is & $1 / 3$ & as & $m$ & 10 & IH & in & III & 10 & $1 x$ & 18 & $w$ & in \\
\hline 1 & 24 & Ist & 131 & in & 20 & In & 27 & an & in & iH & til & if & $2 x$ & 16 & if & 16 & ts & in \\
\hline 1 & 12 & 18 & 10 & $6 x$ & 29 & 20 & 198 & 03 & 18 & 18 & 218 & $2 \pi$ & $2 x$ & IU & 12 & III & 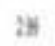 & 20 \\
\hline 1 & 1H & 16 & in & Ift & 18 & $2 x$ & 24 & 028 & 13 & 19. & 24 & 13 & 211 & 16 & 16 & 12 & 18. & 18 \\
\hline 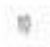 & $2 P$ & (4) & 11 & 19 & $3 n$ & 26 & 21 & m & 13 & ie & 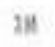 & 28 & 19 & ty & 14 & 18 & 34 & 20 \\
\hline II & 18 & 18 & 17 & Hy & 118 & 28 & 30. & 187 & 19 & III & Alt & 20 & w & 18 & 191 & $n y$ & 19 & 25 \\
\hline $\mathrm{g}$ & $w$ & UI & in & $12 \pi$ & is & $2 \pi$ & 25 & 12 & 18 & vy & 58 & 25 & 12 & ix & 14 & His & 88 & 27 \\
\hline
\end{tabular}

Fuer Notyeas(\$S)

Ahora bien, el programa de pollos de engorde utiliza dos sistemas de crianza, según la publicación Manejo de Pollo de Engorde (Colina y Pérez, 1997):

- Sistema todo adentro, todo fuera: este sistema se refiere a que todos los pollitos son de una misma edad, son iniciados el mismo día y luego de seis semanas, salen a la venta, existiendo un momento en el que no hay aves dentro de las instalaciones. Este sistema rompe cualquier ciclo de enfermedades infecciosas y resulta hasta ahora el más práctico.

- Sistema de crianza múltiple: en este sistema, los pollitos que se encuentran en la granja son de diferentes edades, implica mayor control de enfermedades y aislamiento de aves, además de que cada edad tiene un manejo diferente, lo cual complica la crianza.

Así pues, la elección adecuada del proceso productivo de engorde de pollos responde fundamentalmente a las condiciones higiénicas asumidas por la empresa, así como también a la calidad de los alimentos que consumirán durante el proceso, puesto que si se desea un ave que cumpla con los requerimientos mínimos exigidos, relativos al peso y el tiempo en la conversión de alimentos, se debe ser muy cuidadoso en cada una de las fases que integran este proceso, para así, poder ofrecer productos de excelente calidad al 
consumidor final.

\section{Costos de producción en pollos de engorde}

Los costos de producción en cualquier organización requieren de un tratamiento especial para lograr mantener la empresa en el mercado; éstos deben ser monitoreados constantemente para garantizar la rentabilidad y la ganancia neta de las empresas, que de esta manera pueden ofrecer productos con precios competitivos en el mercado.

Según Gayle (1999), la contabilidad de costos es un elemento fundamental en el proceso productivo; ésta identifica, mide, define, reporta y analiza los diversos elementos de costos. Su principal objeto es comunicar información financiera y no financiera a la administración a efecto de ejercer la planeación, el control y la evaluación, para la toma de decisiones.

Según el Diccionario de la Contaduría General de la Nación (1998) los Costos son erogaciones o causaciones de obligaciones ciertas, relacionadas directamente con los procesos de producción de bienes o prestación de servicios que se recuperan en el desarrollo de la actividad de enajenación. En su acepción más simple y contablemente hablando, Hongren et al. (1996) y Polimeni et al. (1994), definen un costo como un recurso al que se sacrifica o al que se renuncia para alcanzar un objetivo específico. Los costos se miden en unidades monetarias y constituyen el valor que se paga para adquirir bienes y servicios.

En tal sentido, al definir los costos de producción (también llamados costos de fabricación o manufactura) se agregan nuevos elementos; los desembolsos necesarios para mantener un proyecto, línea de procesamiento o un equipo en funcionamiento.

Según el Diccionario de la Contaduría General de la Nación (1998), un costo de producción es una denominación que agrupa las cuentas representativas de las erogaciones asociadas directamente con la elaboración o producción de bienes, o la prestación de servicios de los cuales se obtienen los ingresos. Por su parte, Polimeni et al. (1994), definen los costos de producción como aquellos relacionados con la producción de un artículo y constituyen de acuerdo con Polimeni et al. (1994: 23), Horngren et al. (1996), Gayle (1999), la suma de los costos de mano de los materiales directos, la mano de obra directa y los costos indirectos a la fabricación.

Para Mallo et al. (1998), el concepto económico de costos se ha utilizado en dos 
versiones generales: la primera, en sentido de consumo o sacrificio de recursos de factores productivos, y la segunda en el sentido de costos alternativo o de oportunidad. En tal sentido, Pedersen (1958) citado por Mallo et al (1998), define Costos como el consumo valorado en dinero de bienes y servicios para la producción que constituye el objetivo de la empresa, mientras que para Schneider (1962), citado por Mallo, et al (1998), costos es "el equivalente monetario de los bienes aplicados o consumidos en el proceso de producción".

Para fines de la presente investigación, la definición de costos a utilizar corresponde a la relativa al consumo o sacrificio, puesto que para la fabricación de un determinado producto o para la prestación de servicios, existen insumos o materiales que se transformas con la adición de los medios de producción y la fuerza de trabajo que son factores generadores de costos en bienes o productos terminados o en determinados servicios, aclarando como lo establece Mallo, et al (1998: 33) que todo sacrificio, para que sea costos debe aumentar el valor social del bien al que se aplica; todo sacrificio que no cumpla esta condición debe ser considerado como un despilfarro.

El costo de producción tiene dos caracter ísticas opuestas, que algunas veces no son bien entendidas en los países en vías de desarrollo; la primera es que para producir bienes se debe generar un costo; y la segunda es que los costos deberían ser mantenidos tan bajos como sea posible y/o eliminar los innecesarios. Al analizar éstas características, se evidencia lo siguiente: los costos que implica producir un determinado bien o servicio, se derivan de los factores plasmados en el Gráfico № 2, relativos a la fuerza de trabajo y a los medios de trabajo principalmente; sin obviar, el objeto de transformación que en el caso de engorde los pollos se refiere a los insumos (pollitos bebés), ni los productos y desechos resultantes del procesos, que igualmente generan costos; ante esta situación el granjero incurre en costos para engordar las aves; sin embargo, si su meta es reducirlos existen dos vías o alternativas: a) Al reducir los costos de los insumos necesarios para la fabricación, se afecta la calidad del producto final; y b) al reducir el personal contratado para la cría de los pollos, se reducen costos laborales, en tales circunstancias el granjero se inclina por la segunda alternativa; reducir costos laborales, incrementando la jornada de trabajo de los empleados que aún participan en el proceso; esto constituye una manera de flexibilizar las relaciones laborales y de ahorrar costos laborales.

Los costos de producción presentan varias clasificaciones que se deben analizar; ellas atienden a diversidad de criterios y cada una aplica a condiciones específicas. Autores contemporáneos como Polimeni, et al (1994), Horngren, et al (1996), Gayle (1999) y Hansen y Mowen (1996), establecen que los costos de producción están integrados por: 
Costos de materiales, costos de mano de obra y costos indirectos de fabricación. En tal sentido, se realizará un análisis de los costos de producción.

Los costos de materiales, están relacionados con los principales recursos que se usan en la producción. Los costos de materiales, son aquellos que se transforman en bienes terminados con la adición de mano de obra directa y costos indirectos de fabricación. En esta categoría de costos se encuentran aquellos de adquisición de materiales que con el tiempo se convierten en parte del producto final y a los cuales puede realizarse un seguimiento en forma económicamente factible. El costo de los materiales puede dividirse en materiales directos e indirectos. Los Materiales directos son los que pueden identificarse en la fabricación de un producto terminado, fácilmente se asocian con éste y representan el principal costo de materiales en la elaboración del producto, mientras que los Materiales indirectos están involucrados en la elaboración de un producto y se incluyen como parte de los costos indirectos de fabricación.

Los costos de mano de obra, se refieren al esfuerzo físico o mental empleado en la fabricación de un producto. Los costos de mano de obra pueden dividirse en mano de obra directa e indirecta. Los Costos de Mano de obra directa se refieren a las compensaciones de toda la mano de obra directamente involucrada en la fabricación de un producto terminado y puede asociarse a éste con facilidad, mientras que la Mano de obra indirecta se refiere a aquella involucrada en la fabricación de un producto que no se considera mano de obra directa, por lo cual se incluye como parte de los costos indirectos de fabricación. Porter (1999: 164) establece que los costos de la mano de obra son potentes determinantes de la competitividad.

Los costos indirectos de fabricación también llamados costos generales de producción, son aquellos que, aunque se consideran como parte del objeto de costos, no puede realizarse un seguimiento en forma económicamente factible. Esta categoría de costos se utiliza para acumular los materiales indirectos, la mano de obra indirecta y los demás costos indirectos de fabricación que no pueden identificarse directamente con los productos específicos.

Los costos como variable estratégica de la organización, representan el valor económico de los recursos que la empresa consume en el desarrollo de sus actividades. El análisis de los elementos de los costos de producción: materiales directos, mano de obra directa y costos indirectos de fabricación, es imprescindible en esta investigación. En las empresas avícolas y en particular en las estudiadas, los costos de producción en sus tres elementos incluyen los siguientes aspectos: 
Los costos directos, representado por los materiales directos y la mano de obra directa, se refieren a:

- Los costos de los materiales directos, incluyen básicamente los relativos a los insumos indispensables para la producción, en este caso representado por los Pollitos Bebés necesarios para iniciar el proceso productivo, los medicamentos y alimentos necesarios para realizar la transformación del pollito bebé atendiendo a ciertos requerimientos preestablecidos, para alcanzar un pollo con un peso aproximado de 1.8 a 2.1 Kilogramos.

- Con respecto a los costos de mano de obra, es decir el recurso humano que interviene directamente en la producción, en este caso la cría o el engorde de los pollos, estaría relacionada con el personal obrero, veterinario y cualquier otro personal directamente implicado en la crianza de las aves.

En lo que respecta a los costos indirectos de fabricación o costos generales de producción, las empresas estudiadas lo presentan como otros gastos, y se asumen en este rubro los costos de electricidad, agua, alquiler de la granja, en caso de que sea alquilada, costos por mantenimiento de las instalaciones entre otros; es decir, los costos que indirectamente contribuyen al desarrollo oportuno del proceso, y que por la naturaleza misma de la actividades resulten difíciles de determinar.

Otra clasificación de costos muy utilizada es la de Costos Variables y Costos fijos. Según Horngren, et al (1996:29-30), los Costos variables; son definidos como un costo que cambia en proporción directa al volumen de producción, mientras que los Costos fijos son costos que no cambia a pesar de los cambios en el volumen de producción.

Los costos variables en la cría de pollos de engorde estarían representados por los pollitos bebé, la alimentación y los medicamentos; éstos variarían de acuerdo al número de pollitos bebés que inicien un proceso. Los costos fijos los representarían aquellos factores invariables con el nivel de producción; es decir, aquellos que independientemente del número de pollitos que inicien el proceso van a ser los mismos; ejemplo de ello sería el alquiler de la granja en caso de que sea arrendada y el pago al personal encargado de la crianza de los pollos; todos ellos serían los mismos para 100 pollitos o para 1000.

Desde otra perspectiva, los costos pueden clasificarse en costos primos y costos de conversión; los primeros también son llamados costos directos constituyen los materiales directos y la mano de obra directa. Por lo general, los administradores mantienen 
registros exactos que muestran el costo de los materiales usados para la manufactura de un producto o servicio específico (Gayle, 1999: 33); mientras que los segundos, los costos de conversión, están representados por los costos de mano de obra directa y los costos indirectos de fabricación. Se incurre en estos costos para transformar materiales en productos terminados (Horngren et al., 1996: 43).

Los costos de conversión están referidos al número de unidades que se trabajan y el tiempo que se requiere (minutos), para finalizar la operación individual. Los costos de conversión (mano de obra directa y costos indirectos de fabricación), se compilan para cada operación y después se asignan a todas las unidades.

En lo que respecta al sistema de costeo que emplea este tipo de empresas, analicemos primeramente cada uno de ellos partiendo de los planteamientos de reconocidos autores en el área.

Según Horngren et al. (1996: 97) en los "sistemas de costeo por órdenes de trabajo el objeto de costo es una unidad individual, un lote o servicio de un producto definido, por lo general el producto o servicio está personalizado", es decir están referidos a productos relativamente heterogéneos, mientras que en los sistemas de costeo por proceso se acumulan unidades idénticas o similares de un producto o servicio, este tipo de sistema tiene la particularidad de permitir dividir los costos totales de producir un artículo idéntico o similar entre la cantidad total de unidades producidas para obtener el costo por unidad que se aplica a todas las unidades.

También existe el costeo por operaciones, el cual es un sistema de costeo intermedio para manufacturar bienes que tengan distintos materiales directos, pero operaciones similares de procesamiento. Algunas empresas los denominan sistemas de producción combinados para los cuales se utiliza un sistema de costeo igualmente combinado, es decir un sistema híbrido de costeo, definido por Horngren, et al (1996:656), como mezclas o combinaciones de características, tanto de los sistemas de costeo por trabajo como de costeo por procesos. Los sistemas híbridos de costeo se desarrollan porque existen sistemas híbridos de producción, que constituyen mezclas de la manufactura de órdenes específicas y de la manufactura de producción masiva. En cada operación se utiliza un sólo costo de conversión unitario promedio. Este tipo de sistema de costeo es compatible con la producción de pollos de engorde.

En las empresas avícolas analizadas en los Municipios Mara, La Cañada de Urdaneta y Jesús Enrique Losada, el sistema de costeo predominante, responde a una manufactura de 
producción masiva; es decir, se producen cantidades similares de un solo producto, que requieren un tratamiento similar. En el caso particular de las granjas estudiadas, los pollitos bebés que se constituyen en insumos homogéneos en un $100 \%$, cuando el sistema es "todo adentro, todo fuera", utilizan un sistema de costeo por procesos. Cuando el sistema, es de crianza múltiple, los pollitos que se encuentran en la granja son de diferentes edades, por lo que requerirán de un tratamiento especial, por separado, que puede constituirse en un sistema de producción combinado.

Shank y Govindarajan (1995), establecen que un conocimiento sofisticado de la estructura de costos de una empresa, puede ser de gran ayuda en la búsqueda de ventajas competitivas sostenibles y es lo que ellos denominan gerencia estratégica de costos. Por esta razón, en esta investigación se pretende analizar la estructura de costos de las empresas del sector avícola ubicadas en los municipios Mara, La Cañada de Urdaneta y Jesús Enrique Losada.

Pimpollo, Vilva, Avícola de Occidente, Protinal, Proave, Souto, y Criazuca, son empresas que operan en la zona, con niveles de integración vertical y escalas de producción diferentes, lo que supone costos de producción diferentes. Sin embargo, la escasa variabilidad en los costos unitarios los hace suficientemente homogéneos como para poder comercializar los pollos a los precios regulados por el gobierno.

Los costos de producción de pollos de engorde en las empresas objeto de estudio dependen en primer lugar de las condiciones impuestas por la industria matriz, de la integración a la cadena de valor (Interacción Vertical), y en segundo lugar al volumen de producción (escala) y del tamaño de la granja. La capacidad de la granja implica costos fijos que afectan los costos de producción, especialmente cuando no se trabaja a plena capacidad.

La cadena de valor en cualquier empresa, es el conjunto interrelacionado de actividades creadoras de valor que se extienden durante todos los procesos; van desde la consecución de fuentes de materias primas hasta que el producto terminado se entrega finalmente en las manos del consumidor. Este enfoque es externo a la empresa, considerando a cada compañía en el contexto de la totalidad de la cadena de actividades creadoras de valor de la cual la empresa es sólo una parte (Shank y Govindarajan, 1995: 16).

En las industrias avícolas, las actividades que integran la cadena de valor o cadena productiva pueden resumirse en las siguientes: 1) Planta de Alimentos; 2) Cría y Recría (Levante); 3) Reproductores de huevos fértiles; 4) Incubadora (Nacimiento y Selección); 
5) Engorde (Granjas propias y alquiladas); 6) Beneficio (Matadero); 7) Mercado y Comercialización (Ver Diagrama 3).

Es importante enfatizar que esta investigación se aboca solamente al an álisis del proceso de engorde de los pollos, en granjas que pueden ser propias o alquiladas. El resto de los procesos no son objeto de análisis en esta instancia, aunque indudablemente inciden en el costo de producción.

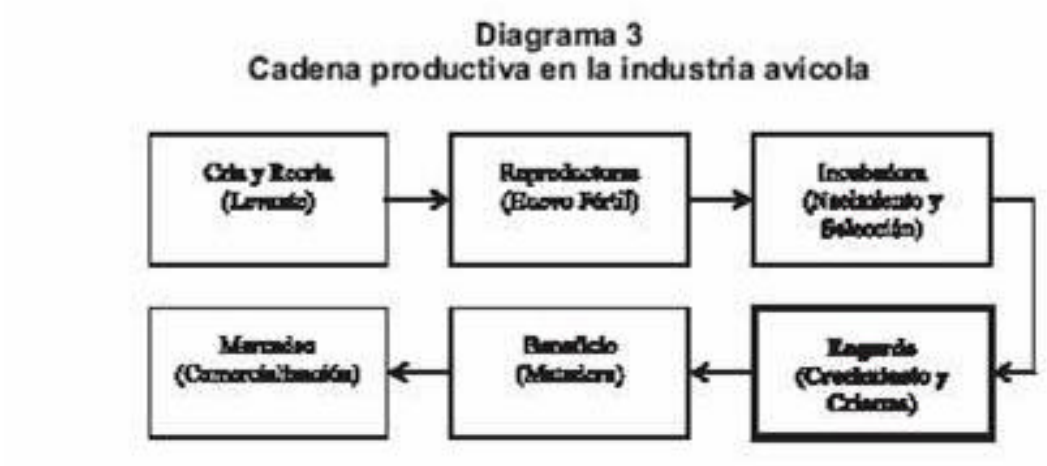

Fuen be: Elaboración Propia (Orozca y Meledn, 2004)

Ahora bien, el proceso para determinar costos de producción tanto en granjas propias como alquiladas, debe cumplir con los siguientes requisitos, que constituyen soportes de costos para las granjas analizadas:

1. Entrega de nota de envío de pollitos bebés, de la incubadora a la granja.

2. Nota de entrega de vacunas y medicinas de la empresa a la granja.

3. Nota de entrega de tickets de Romana por envío de alimentos.

4. Registro del consumo de alimento

5. Mortalidad de aves.

6. Orden de entrega de pollos al matadero o planta de beneficio.

7. Cálculo de pago al granjero según una tabla preestablecida (tabulador).

Una estructura de costos, típica de la zona, contempla los siguientes rubros: Pollitos bebés, Alimentación, Vacunación, Medicinas, Fumigación, Pago al granjero, Otros gastos. La Tabla 2 y Gráfico 1, expresa la estructura de costos construida con base en los datos suministrados por los gerentes de cada una de las granjas encuestadas. La información relativa a costos al cierre del 31 de diciembre del año 2003, es la siguiente:

El rubro de alimentación representa, en promedio, un $71.20 \%$ del costo total del proceso de engorde; este costo varía entre $68.00 \%-75.05 \%$ en las granjas estudiadas, lo que da 
una idea de la importancia del rubro alimenticio en una actividad de este tipo. Debido a que el alimento representa el renglón más costoso en la producción de pollos de engorde y por la variación en la conversión de alimentos de acuerdo a la edad, sexo y peso del ave, lo mejor es que se comprendan estas variaciones y sus implicaciones en el negocio. Según la Cooperación Económica Asiática (1999: 29), en todas las economías, el costo de alimentos para animales a nivel de los pollos de engorde es el mayor costo dentro de la producción.

El costo de producción de un kilogramo de pollo es muy variable de granja en granja, de una estación a otra y de un país a otro. La importancia decisiva del alimento balanceado en la producción avícola puede evidenciarse en la elevada participación de este insumo en la estructura de costos de producción. Este hecho, transforma a la industria avícola en un sector dependiente de la variación de precios de los alimentos balanceados.

Los costos por la adquisición de los pollitos bebés necesarios para dar inicio al proceso de engorde, se encuentra entre el 13 y $14 \%$ del costo total de producción; la cría de éstos pollitos requiere también del alimento balanceado; esto significa que cerca del 81.90 al $87.76 \%$ del costo total de producción de pollos de engorde dependen del valor de un insumo determinante: el alimento balanceado.

Tabla 2

Estructura de costos de la cria de pollos de engorde Municipios Mara, la Cañada de Urdaneta y Jesús Enrique Losada, estado Zulia (2003)

\begin{tabular}{|c|c|c|c|c|c|c|c|}
\hline & Granju I & Grunja 2 & Granja 3 & Grunju-4 & Granjas & Granja 6 & Granja 7 \\
\hline Elvmenter de Cortes & $\%$ & $\%$ & $\%$ & $\%$ & $\%$ & 96 & $\%$ \\
\hline Folliwe Bete & 14,00 & 12.71 & 13,42 & 14,00 & 14,40 & 13,90 & 1205 \\
\hline Alireknte & 72,29 & 75,05 & 60,08 & 71,08 & 73,00 & $\leqslant 8,00$ & 70,00 \\
\hline Vhetemacien & 0,39 & 0.40 & 0.40 & 0.50 & 0,30 & $0,+0$ & 0.57 \\
\hline Motsitus & 0.14 & 0.15 & 0,25 & 0,30 & 0,14 & 0,20 & 0,25 \\
\hline Feniescion & 0,12 & 0,13 & 0,15 & 0,18 & 0,10 & 0,15 & 0,20 \\
\hline Powo al gantiene & 9.010 & $k f 0$ & 8,40 & 10,00 & 9,00 & $x \infty$ & 8.70 \\
\hline Otros & $3.4 t$ & 287 & 8,23 & 4,02 & 3,06 & 8,45 & (x) 30 \\
\hline TOTAL. & 160,00 & 100.90 & 160,00 & 100,00 & 100,00 & 160,00 & 100.90 \\
\hline
\end{tabular}

Funns: Baboracón propà, con irforradón suminis rada por las grarjas estudiadas 
Gráfico 1

Distribución de los costos de crianza de pollos de engorde Municipios Mara, La Cañada de Urdaneta y Jesús Enrique Losada, estado Zulia (2003)

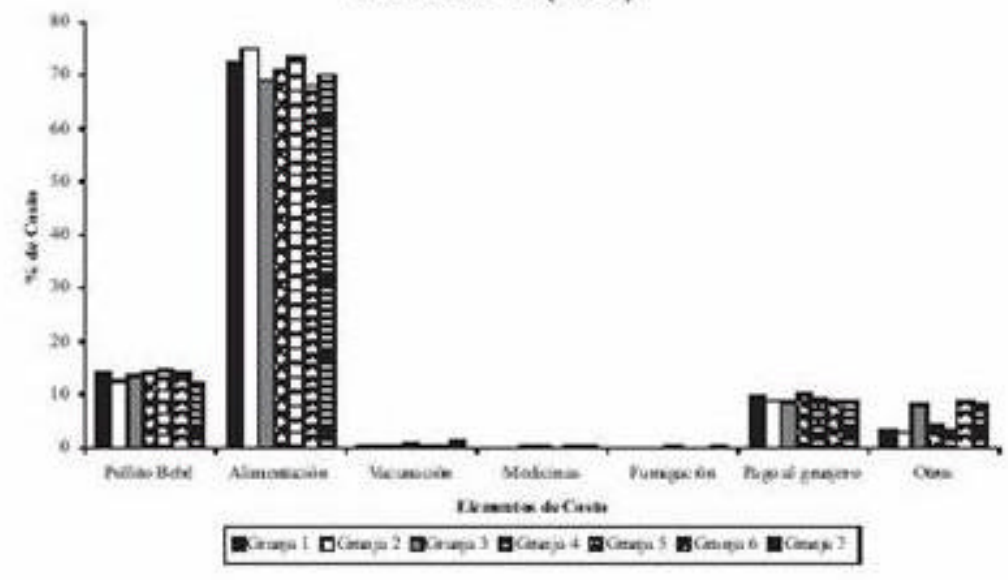

Fuente: Elaboradon propa con informacón suminstrafa por las granjas estudiadas

Los costos de los alimentos para animales es el costo más importante en esta etapa de la cadena productiva del sector avícola, no sólo por el costo de los alimentos sino también por el impacto del costo de los alimentos para las reproductoras sobre el costo de los pollitos bebés de un día de nacido.

Por otra parte, en lo que respecta al costo unitario de la mano de obra y la productividad de la misma en la actividad de levante representa el segundo costo más importante, aclarando que el pago de los criadores variará dependiendo la estructuración de la industria y de las condiciones locales. Los demás costos son relativamente pequeños en comparación con los ya analizados.

\section{Conclusiones}

Las medidas económicas establecidas por el gobierno, así como también las importaciones de pollo realizadas, han golpeado fuertemente al sector, sin embargo, el ejecutivo lo que ha buscado es garantizar la seguridad alimentaria con la colocación de este producto en el mercado. A mediados del 2004, se ha regularizado en gran medida la producción avícola, pero muchos de los productores no han acatado los precios regulados, argumentando que tales precios no cubren sus costos de producción, los cuales representan un factor clave para el éxito de este tipo de empresas.

Las estructuras de costos de las granjas analizadas en esta investigación, arrojó como resultado que en promedio el $71.20 \%$ de los costos de producción está representado por 
los costos de alimentación, seguido por la adquisición de los insumos (pollitos bebés) con un $13.50 \%$ y el pago al personal que interviene en las operaciones llevadas a cabo en las granjas con un $9.05 \%$.

Los altos costos de los rubros de alimentación y de la adquisición de los pollitos bebés como insumos para iniciar el proceso de engorde de las aves, constituyen las bases de la integración vertical de la industria avícola; en esta integración, las empresas grandes ejercen control sobre empresas de menor tamaño, en este caso las granjas criadoras de los pollos, quienes aceptan las condiciones de crianza, higiene y alimentación entre otras impuestas por la empresa que controla la red.

La importancia de adquirir los pollitos bebés a tiempo para comenzar el proceso productivo, así como la necesidad de garantizar los alimentos en el momento preciso y a precios que permitan obtener un margen de ganancias, ha llevado a las empresas a integrarse verticalmente en redes de proveedores; con esto logran garantizar una fuente de aprovisionamiento importante para su producción. La integración vertical en redes de distribuidores también es importante en este tipo de productos, puesto que el carácter de perecedero y las condiciones en las cuales se debe mantener implica necesariamente una integración vertical con los distribuidores de los productos terminados, quienes harán llegar en las mejores condiciones el producto final a los consumidores finales. Controlar las actividades en cada una de las etapas de la cadena productiva para obtener productos terminados con la mayor calidad y a precios competitivos son los resultados de la integración en red de la industria avícola nacional.

\section{Notas}

* Este artículo es resultado del Programa de Investigación "Presupuestos y Costos de Producción" financiado por el Consejo de Desarrollo Científico y Humanístico (CONDES).

1. FAO, por sus siglas en inglés.

\section{Referencias Bibliográficas}

1. Asamblea Nacional (1999), Constitución de la República Bolivariana de Venezuela.

2. Barreiro, Raquel (2003), \$180 millones para importar alimentos decembrinos. CONSUMO / Ministerio de Agricultura abrirá la taquilla navideña. El Universal http: //www.ciec.org.ve/prensa/titularesh4.asp?eje=30\&page=206 Fecha de consulta: 
$14 / 01 / 04$.

3. Buffa, Elwood S. y Sarin, Rakesh K. (1992), Administración de la Producción y de las Operaciones. Limusa Grupo Noriega Editores. Primera Edición. México. Pág. 939.

4. Cadivi (2003), I mportaciones. http://www.cadivi. gov.ve/index.html Fecha de consulta: $19 / 08 / 2003$

5. Carvajal Centeno, Ilich (2002), ¿Venezuela, Agricultura de Puerto? Panorama, Pág 5. Fecha: 08-07-02.

6. Chase, Richard; Aquilano, Nicolas y Jacobs, Robert (2000), Administración de Producción y Operaciones. McGraw-Hill Interamericana, S.A. Octava Edición. Colombia. Pág. 885.

7. Colina, Argenis y Pérez Carlos (1997), Manejo de Pollo de Engorde Guía de Estudio. Dpto. de Producción Animal. Cátedra de Producción y Patología Aviar. Facultad de Ciencias Veterinarias. Universidad del Zulia. Maracaibo-Venezuela.

8. Contaduría General de la Nación (1998), Diccionario de términos de contabilidad pública. República de Colombia, Sub contaduría de Consolidación e investigación.

9. Cooperación Económica Asiática (1999), La Cadena Productiva de la Industria Avícola. http://www.pcbec.org/publications/poultry/poultry2.pdf. Fecha de consulta: 3009-2004.

10. Coriat, Benjamin (1995), Pensar al Revés. Trabajo y organización en la empresa japonesa. Siglo Veintiuno Editores. 2da Edición. México.

11. Davis, Marck M.; Aquilano, Nicholas J. y Chase Richard B. (2001), Fundamentos de Dirección de Operaciones. Tercera Edición. McGraw-Hill. España.

12. Diccionario Pequeño Larousse Ilustrado (1997), Coedición Internacional, Dinamarca - Argentina - México. Ediciones Larousse.

13. Fernández, Esteban; Avella, Lucia y Fernández, Marta (2003), Estrategia de Producción. McGraw-Hill. Madrid, España. 
14. Gamboa Cáceres, Teresa; Arellano Rodríguez, Madelein y Nava Vásquez, Yuneska (2003), Actores y fines de las estrategias empresariales. Una reflexión desde las pequeñas y medianas empresas. Revista Visión Gerencial del Centro de Investigaciones y Desarrollo Empresarial (CIDE). Año 2 No. 1. Vol. 1 Enero-Junio. Universidad de los Andes. Páginas 28-39. Mérida, Venezuela.

15. Gamboa, Teresa y Naveda, Oscar (1999), Propuesta de un Modelo de Evaluación Institucional por Procesos y Resultados. La Universidad del Zulia. Vicerrectorado Administrativo. Centro de Estudios de la Empresa (FCES).

16. García Cantú, Alfonso (1995), Productividad y Reducción de Costos: para la peque ña y mediana industria. Editorial Trillas. México.

17. Gayle Rayburn, Letricia (1999), Contabilidad y Administración de Costos. McGraw-Hill Interamericana Editores. Sexta Edición. México. Pág. 971.

18. González, Mario (2003), Plan Avícola y Agropecuario recibirá 170 millardos. http://www. ciec.org.ve/prensa/titularesh4.asp?eje=30\&page $=381$ Venpres. Fecha de consulta: 14/01/04

19. Hansen, Don y Mowen, Maryanne (1996), Administración de Costos. Contabilidad y Control. International Thomson editores, S.A. de C.V. México. Pág. 502.

20. Hernández, Katiuska (2003), Agroindustria solicita celeridad en otorgamiento de divisas. El Nacional. Http:// www.ciec.org.ve/prensa/titularesh4. asp? eje=30\&page $=131$. Fecha de consulta: 14/01/04.

21. Horngren, Charles; Foster, George y Datar, Srikan (1996), Contabilidad de Costos. Un enfoque gerencial. Octava edición. Prentice Hall Hispanoamericana. México. Pp. 970.

22. Mallo, Carlos; Kaplan, Roberts; Meljem, Sylvia y Jiménez, Carlos (2000), Contabilidad de Costos y Estratégica de Gestión. Prentice Hall I beria. Madrid. España. Pág. 768.

23. Ministerio de Agricultura y Cría (1997), VI Censo Agrícola. Venezuela.

24. North, Mack y Bell, Donald (1993), Manual de Producción Avícola. Cuarta Edición. Editorial El Manual Moderno, S.A. México. 
25. Organización de las Naciones Unidas para la Agricultura y la Alimentación, FAO (2003a), Programa Especial para la Seguridad Alimentaria. Consulta: 08/09/03 http://www.fao. Org/spfs/index.asp?lang=es

26. Organización de las Naciones Unidas para la Agricultura y la Alimentación, FAO (2003b) Objetivos y planteamiento. Consulta: 08/09/03 http://www.fao. org/spfs/objectives_es.stm\#top

27. Perozo, Maira Cecilia (2003), Seguirán habiendo dólares para la importación de alimentos. Panorama - Política, lunes 5 de mayo. Año 89, № 29.762 Página 2-4. Maracaibo, Venezuela.

28. Polimeni, Ralph S.; Fabozzi, Frank J.; y Adelberg, Arthur K. (1994), Contabilidad de Costos. Conceptos y Aplicaciones para la Toma de Decisiones Gerenciales. Tercera Edición. McGraw-Hill Interamericana, S.A. Santa Fe de Bogotá. Colombia.

29. Porter, Michael (1999), Ser Competitivo. Nuevas aportaciones y conclusiones. Ediciones Deusto. México.

30. Rodríguez, Guillermo; Balestrini, Solange; Balestrini, Sara; Meleán, Rosana y Rodríguez, Belkis (2002), Análisis Estratégico del Proceso Productivo en el Sector Industrial. Revista de Ciencias Sociales. Volumen VIII. No. 1. Enero-Abril. Páginas 135156. Universidad del Zulia. Venezuela.

31. Shank, John y Govindarajan, Vijay (1995), Gerencia Estratégica de Costos. La nueva herramienta para desarrollar una ventaja competitiva. Primera Edición. Grupo Editorial Norma. Colombia. Pág. 340.

32. Sipper, Daniel y Bulfin Jr. Robert (1998), Planeación y Control de la Producción. Editorial McGraw-Hill. Mexico. Páginas 657.

33. Velasco, Julia (1997), I mpacto de las reformas económicas sobre la industria avícola. Trabajo de ascenso Facultad de Ciencias Veterinarias. LA Universidad del Zulia. Maracaibo-Venezuela. 